\title{
Entwicklungen und Perspektiven in der Dialektologie des Deutschen: Einige Schlaglichter
}

\author{
Jürgen Macha (Münster)
}

\begin{abstract}
This article is divided into four sections. Section one draws a sketch of the developments in German dialectology during the past 30 years. Section two establishes the distinction between the description of dialects focusing on the linguistic system, dialect sociology referring to usage and evaluation, and dialect pragmatics. Section three proposes research perspectives within the framework of a 'new dialectology'. The concluding section four introduces an empirical project in co-operation of several universities (Münster, Bonn, Hamburg, Bielefeld, Potsdam, Frankfurt/Oder) designed to research into the current linguistic situation in northern Germany.
\end{abstract}

Die folgenden Ausführungen gliedern sich in vier Abschnitte. Teil 1 behandelt allgemeine Entwicklungen der deutschen Dialektologie im letzten Drittel des 20. Jahrhunderts. In Teil 2 schlage ich eine Untergliederung der synchronen Dialektologie bzw. Nonstandard-Forschung nach Erkenntniszielen vor. Teil 3 enthält sehr subjektiv geprägte Vorschläge für Forschungszugriffe. In Teil 4 schließlich werden Grundzüge eines kooperativ angelegten empirischen Projektes zur Durchleuchtung der Sprachsituation in Norddeutschland skizziert.

\section{Entwicklungen in der Dialektologie seit den 60er Jahren}

1967 schrieb Hermann Bausinger: "Wer die heutigen sprachlichen Verhältnisse untersucht, gerät in ein Feld von Übergängen, Ausgriffen, Zwischenformen, Unsicherheiten. Die Frage stellt sich, wo in dieser Situation vernünftigerweise Beobachtung und Analyse anzusetzen haben." (Bausinger 1967: 309f.).

Seit dieser Zeit haben ganz verschiedene Entwicklungen Platz gegriffen, wobei sich eine rasche Aufeinanderfolge unterschiedlicher erkenntnisleitender Vorstellungen und eine fortschreitende Auffächerung der Interessen ergeben hat. Den gemeinsamen theoretischen Fluchtpunkt bildete freilich die Abkehr von einer Auffassung, derzufolge es der Disziplin lediglich und ausschließlich um die Erforschung der arealen Verbreitung von Sprache zu gehen habe. Eine solche einseitige Auffassung von Dialektologie als Dialektgeographie, oder wie Scheuringer anmerkt, von Dialektologie als 'Topolektologie' (Scheuringer 2000: 431) kann durch die Forschungsgeschichte seit 1970 als revidiert und ergänzt gelten. 1985 äußerte 
William G. Moulton aus Anlass des Erscheinens des Dialektologie-Handbuchs und angesichts der Vielfalt neuartiger Forschungsperspektiven in einer Rezension: "... wie hat sich doch die Disziplin Dialektologie gerade in den letzten Jahren ausgeweitet und bereichert!" In ihrem instruktiven Sammelband 'Dialektologie des Deutschen. Forschungsstand und Entwicklungstendenzen.' von 1994 gliedern Peter Wiesinger und Klaus J. Mattheier das Forschungsgebiet der Dialektologie in sechs Arbeitsfelder auf (Mattheier/Wiesinger 1994: IX-XI).

1. Dialektbeschreibung

2. Dialektgeographie

3. Dialektsoziologie

4. Historische Dialektologie

5. Wissenschaftsgeschichte der Dialektologie

6. Computerdialektologie

Die Bereiche 4. und 5. bilden qua Diachronie eigene Forschungsbereiche, die hier vernachlässigt werden können, ungeachtet ihrer Notwendigkeit und ihres wissenschaftlichen Reizes. Punkt 6. ist schon in der Mattheier/Wiesinger-Konzeption nicht als eigenständiges Forschungsfeld konzipiert, sondern eher als methodisch-technisch unterstützend für die anderen aufgeführten Zugriffe gedacht (cf. XI).

\section{Untergliederungen synchroner Dialektologie und Nonstandardforschung}

Angesichts gerade auch von Entwicklungen, die im letzten Jahrzehnt gegriffen haben, möchte ich für eine 'synchrone Dialektologie', die im weiteren Sinne auch als 'NonstandardForschung' begriffen wird, folgendes kleine Schema zur Orientierung vorschlagen:

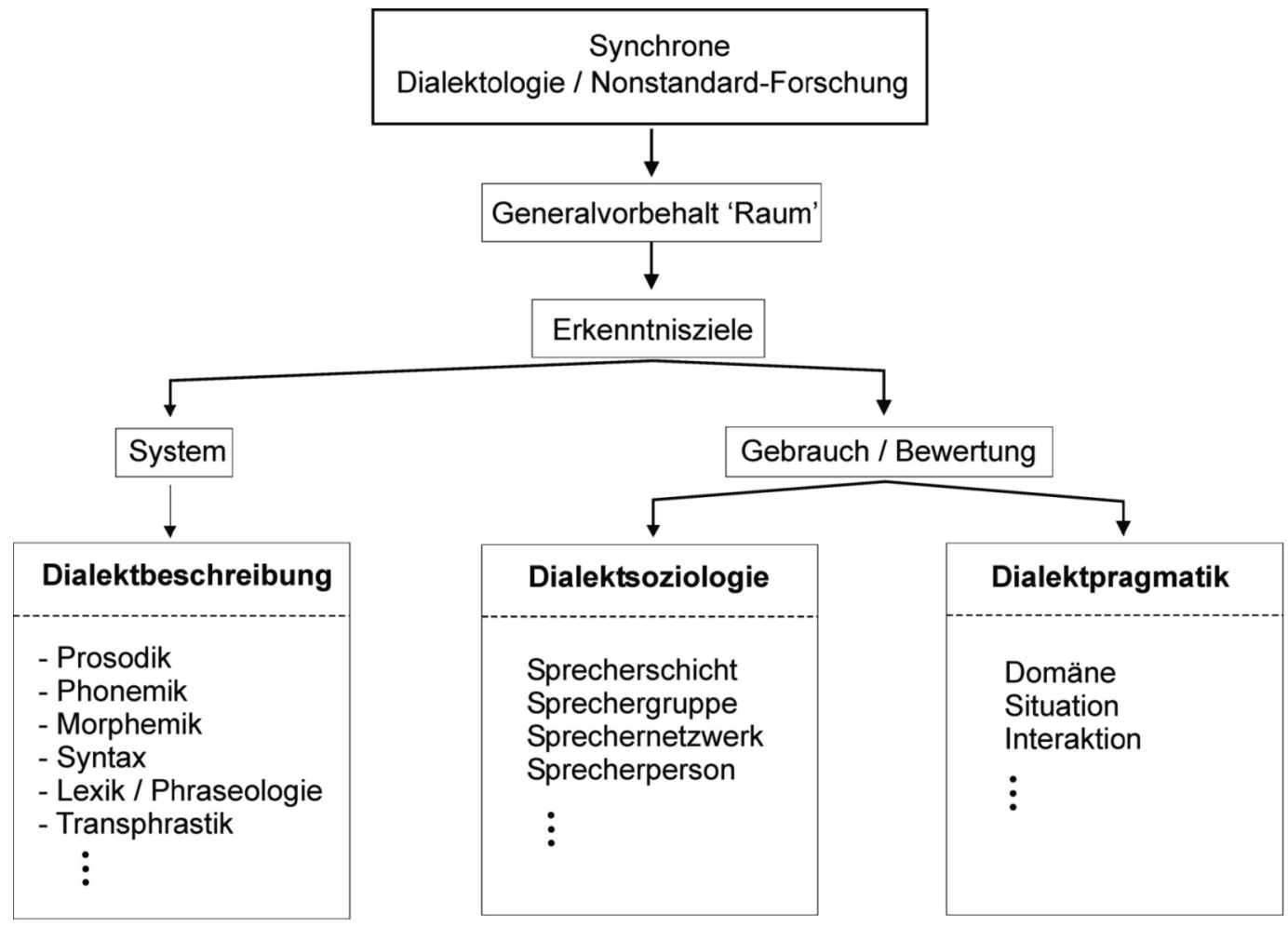


Mit einer etwas anderen Akzentuierung lässt sich auch sagen: Unter dem übergeordneten Aspekt 'Raum' werden die drei Dimensionen 'Sprache als System', 'Sprache als Ausdruck sozialer Verhältnisse' und 'Sprache als soziales Handeln' in den Blick gerückt. Dieses kolossal vereinfachende Schema hat einen Vorteil: Es ermöglicht die Verortung einer Reihe von Ansätzen. Es hat jedoch auch eine Menge Nachteile: So wird die vielfach gegebene Verknüpfung der Erkenntnisziele nicht recht sichtbar, man könnte natürlich Verbindungspfeile o.ä. einzeichnen, ohne dass jedoch den Möglichkeiten angemessen Rechnung getragen würde. Auch der ganze Bereich einer 'Perzeptionsdialektologie' - man denke an Herrgen/Schmidt und ihre 'Hörerdialektalität' (Herrgen/Schmidt 1985) - wird hier nicht explizit berücksichtigt. Andererseits wird m.E. eine prinzipielle Eigenart dialektologischer Forschung herausgestellt: Alle Untersuchungen operieren unter dem Generalvorbehalt 'Raum' und nehmen damit einen grundlegenden Differenzierungsfaktor zum Ausgangspunkt. Vom Denkansatz einer nicht-korpusgestützten Introspektions-Linguistik unterscheidet sich solche Forschung also nicht zuletzt dadurch, dass - bedingt durch die empirisch fundierte Datenbasis - stets 'sprachräumliche Differenzierungen' mitbedacht werden (müssen), bis hin zum Chaos. Die Annahme einer 'standarddeutschen Artikulationsbasis', wie sie beispielsweise Peter Eisenberg in einem münsterischen Vortrag unter Bezug auf eine zunehmend einheitlicher werdende sprachliche Primärsozialisation formuliert hat (Hörbeleg, J.M.), ist eine für manche Aufgaben sinnvolle Idealisierung. Sie erscheint dem empirisch arbeitenden Dialektologen indes fast schon gefährlich artifiziell.

\subsection{Dialektbeschreibung}

Schaut man innerhalb des Großgebietes 'Dialektbeschreibung' auf einzelne Teilbereiche, so sind die Gewichte einer Beschäftigung mit Dialekt bzw. Nonstandard in jüngerer Zeit nicht wenig verschoben worden. Festzustellen ist in diesem Zusammenhang, dass die Erforschung der Suprasegmentalia aus ihrem Schattendasein herausgetreten ist: Die regional verschiedenen konstitutiven Merkmale des Sprechens rücken in jüngerer Zeit stark ins Blickfeld (vgl. Schmidt 1986, Auer/Gilles/Peters/Selting 2000). Dagegen sind die traditionell besonders gepflegten Untersuchungen phonetisch-phonologischer Merkmale - laut Wiesinger - relativ gesehen eher rückläufig (vgl. Wiesinger 1994: 19f.). Es ist allerdings zu berücksichtigen, dass die Lautlichkeit in vielen 'gemischten' Zugriffen durchaus eine wichtige Rolle gespielt hat, man denke etwa an die Stadtsprachenuntersuchungen der 90er Jahre (vgl. etwa Basel u.a., dazu Niebaum/Macha 1999: 157f.). Zudem dominiert dieser Forschungsaspekt nach wie vor in den verschiedenen Sprachatlasunternehmungen im Süden und in der südlichen Mitte des deutschen Sprachraums (z.B. bei den bayerischen Atlanten oder beim Mittelrheinischen Sprachatlas, zu letzterem Niebaum/Macha 1999: 120-125). Über Untersuchungen zur Nonstandard-Lexik lässt sich Ähnliches sagen: In verschiedenen Atlanten ist den entsprechenden Phänomenen umfassend Rechnung getragen worden (vgl. Eichhoff 1977ff., dazu Niebaum/Macha 1999: $132 \mathrm{ff.),} \mathrm{gezielte} \mathrm{Einzelstudien} \mathrm{lassen} \mathrm{sich}$ meines Wissens jedoch weniger finden. Eine Ausnahme bilden neuere Arbeiten, die sich regionalspezifischer Phraseologismen angenommen haben. Hier werden - z.B. von Elisabeth Piirainen - neue interessante Wege beschritten. 
Bis in die achtziger Jahre galten Arbeiten zur Morpho-Syntax und zur Syntax als 'Stiefkinder der Dialektbeschreibung' (vgl. Wiesinger 1993: X). Sieht man von Beate HennMemmesheimers Habilitationsschrift (1986) ab, so hat die Hinwendung zu den entsprechenden Erscheinungen erst um 1990 stärker eingesetzt. In der Folgezeit entstanden diesbezüglich freilich zunehmend ambitionierte Studien, die bisher vernachlässigte Themen in den Blick nehmen und empirisch erkunden. Auch die Zusammensetzung der Beiträge der Freiburger Tagung von 2004 lässt erkennen, dass mit der rezenten Syntaxforschung Lücken der herkömmlichen Dialektgeographie (gerade für den Raum der Schweiz) gefüllt werden.

\subsection{Dialektsoziologie und Dialektpragmatik}

Im Folgenden geht es um Erkenntnisziele, die mit Dialektalität in ihrer gesellschaftlichen Verschränkung verbunden sind. Dabei erweist es sich als schwierig, die recht disparate Fülle der verschiedenen Ansätze einigermaßen plausibel zu bündeln und zu etikettieren. Sowohl von den theoretischen Prinzipien her als auch bei den verwendeten Methoden tut sich eine große Vielfalt auf. Möglicherweise hilft hier ein kurzer Blick auf die Forschungsgeschichte:

Wie bereits angedeutet, ergaben sich im letzten Drittel des 20. Jahrhunderts folgenreiche Weiterungen der Dialektologie im Blick auf die Berücksichtigung sozialer und kommunikativer Zusammenhänge. Wenn man so will, haben wir es mit zwei 'Wenden' zu tun: die erste soziolinguistische Wende liegt um 1970, die zweite pragmatische oder pragmalinguistische etwa gegen 1980. Hängt man das Ganze terminologisch nicht ganz so hoch, so kann man auch sagen: Mit zeitlicher Versetzung sind seit 1970 zwei theoretische Stränge aus der Reflexion über Regionalsprache intensiv ausgebaut und empirisch überprüft worden, die bis dahin zwar nicht im Zentrum des Interesses gestanden haben, die aber spätestens seit Philipp Wegener 1880 wissenschaftlich erkannt waren. Klaus J. Mattheiers Monographie: Pragmatik und Soziologie der Dialekte von 1980 markiert, inhaltlich wie zeitlich, exakt die disziplingeschichtlich vollzogenen Weichenstellungen. Dieses Buch fundierte als Zusammenfassung eine neue Sicht auf die Regionalsprachen, auf ihre Verwendung und ihre Bewertung, wobei in zunehmendem Maße sozialwissenschaftliche Argumente und Modellbildungen relevant wurden.

Zur selben Zeit begann sich freilich an anderer Stelle der Zunft ein leises Unbehagen zu artikulieren, das mit dem implizierten Menschenbild eines 'homo sociologicus' zusammenhängt. 1976 etwa konstatierte Wolfgang Steinig in recht apodiktischer Manier: "Der Faktor 'Individuum', verstanden als individualpsychologische Variable, die Verhalten und damit sprachliches Verhalten beeinflusst, muss in einer soziolinguistischen Untersuchung unberücksichtigt bleiben." (Steinig 1976: 115) 1985 beschäftigte sich dann Heinrich Löffler beim Göttinger Germanisten-Kongress mit herkömmlichen und zukünftigen Tätigkeitsbereichen der Dialektologie. Er kam zu dem Schluss: "Das neue Aufgabenfeld der nachsoziolinguistischen Dialektologie ist [...] die deutsche Sprachwirklichkeit und ihre Sprecher. 'Sprecherdialektologie' wäre vielleicht ein vorläufiger Name." (Löffler 1986: 239) Bei der gleichen Veranstaltung hielt ich einen Vortrag über "Die Bedeutung individueller Variation", mit dem der Gültigkeitsanspruch einiger soziolinguistischer und 
pragmalinguistischer Annahmen als forschungsleitender Paradigmen angekratzt werden sollte. Es blieb auf einige Zeit beim 'Ankratzen', in der Folgezeit kristallisierten sich freilich Kritiklinien heraus, deren Fluchtpunkt in der Relativierung eines 'Modells vom determinierten Sprecher' liegt (vgl. die Ausführungen zum individuenzentrierten Ansatz bei Bürkli 1999: 1219). Zwei Aspekte sind in diesem Zusammenhang besonders theorierelevant: Sprache und Sozialität sowie Sprache und Situativität.

Ad 1: Lange Zeit hindurch ging man in der empirischen Dialektforschung vom Postulat aus, dass die Sprache des individuellen Exemplars einer Gruppe bzw. 'Gattung' die Sprache eben dieser Gruppe bzw. 'Gattung' generell und umfassend zu repräsentieren vermag. Wenn z.B. Vertreter der 'Umfrage-Dialektologie' im 19. Jahrhundert wie Georg Wenker oder noch die Wortatlas-Autoren in den dreißiger Jahren fest überzeugt waren, die Mundart eines Ortes sei via Auskunft über ein einziges sprechendes Individuums abrufbar, so steckt eine kräftige Unterstellung dahinter. Reiner Hildebrandt fasst rückblickend diese Hauptprämisse der DWAArbeit zusammen: "Die Auskünfte einer Gewährsperson stehen stellvertretend für das ganze Dorf und meist auch die ganze Stadt ..." (Hildebrandt 1968: 153) Die dabei behauptete ideale Homogenität ist wenn schon nicht märchenhaften, so doch sicherlich vormodernen Zuständen zuzuordnen: Nur solange man begründet annehmen kann, dass Individuen in weitgehend geschlossenen, traditionalen Gemeinwesen mit gemeinsamem kulturellem Besitztum, inklusive Sprache, zusammenleben, solange lässt sich die These von einer homogenen Ortssprache halten, die das einzelne Subjekt reproduziert. In der soziolinguistischen Dialektologie hat man nun dieses prämoderne Muster auch auf die Binnendifferenzierung von Gemeinwesen angewandt, d.h. die Zugehörigkeit eines Individuums zu einer sozialen Formation in einem Ort präfiguriert dasjenige, was bei ihm an Sprachkompetenz und Sprachbewusstsein zu erwarten ist. Ein solches Modell setzt indes eine hochgradig ständisch organisierte Gesellschaft mit stabilen sozialräumlichen Milieus voraus, in der Durchlässigkeit und Mischung keine Rolle spielen. Wenn aber - wie es für die heutige Zeit zu konstatieren ist - eine umfassende Diversifizierung und Individualisierung von Lebenslagen und Lebenswegen eintritt, dann verändern sich auch die sprachliche Ausstattung und der sprachliche Möglichkeitsraum des Einzelnen in erheblichem Ausmaß.

Ad 2: Wie angedeutet, lässt sich die zweite Determinationsannahme als 'pragmatisch' bzw. 'pragmalinguistisch' bezeichnen. Die Ausprägung individuellen Sprechens hängt danach wesentlich von den situativen Bedingungen ab, unter denen es realisiert wird. Diese vordergründig triviale Aussage wird bedeutsam, wenn man sich anschaut, wie sie inhaltlich gefüllt ist. Zwei Situativitäts-Auffassungen sind für soziodialektologische Fragestellungen in besonderem Maße folgenreich gewesen. Das erste Konzept bündelt sogenannte 'kongruente Situationen' zu 'Domänen' und postuliert die Abhängigkeit individuellen Sprechens von den auf diese Weise gebildeten 'Domänen' bzw. Lebensbereichen (vgl. Fishman 1975). Man eruiert - oft mit den Methoden der Demoskopie - das Sprachverhalten 'in der Familie, im Kontakt mit Freunden, bei der Arbeit' usw. An diesem Verfahren lässt sich massive Kritik üben. Der Sprachgebrauch in der 'Familie' als einer bedeutsamen lebensweltlichen Domäne ist in der heutigen Zeit und in manchen Regionen Deutschlands durchaus mehrdimensional und 
kompliziert. SprecherInnen differenzieren u.U. zwischen familiären Ansprechpartnern und setzen beispielsweise Dialekt gegenüber dem Ehepartner, intendierte Standardsprache jedoch gegenüber den Kindern ein. Die Familie ist dann zwar eine Kommunikationsgemeinschaft, jedoch keine Sprachgemeinschaft mehr in dem Sinne, dass ihre Mitglieder nur eine einzige Sprache benutzen würden. Während das Domänenkonzept eine sektorielle Gliederung gesellschaftlicher Lebensbereiche zugrunde legt, unterscheidet das Formalitäts-Konzept Situationen nach ihrem atmosphärischen Gehalt. Im Formalitäts-Konzept wird die Vielfalt sozialer Situationen, aus denen sich Lebensabläufe bilden, reduziert und in eine Zweiteilung umgesetzt. Die Definition von Situationstypen erfolgt nach den Kriterien 'formal' versus 'informell'. Das sprechende Individuum reagiert in seiner Sprachwahl auf die atmosphärischen Vorgaben; es erscheint als ein janusköpfiges Wesen mit zwei SprachgebrauchsPhysiognomien, das sich je nach Situationstyp verschieden zeigt, also in formalen Situationen eher Standardsprache spricht, in informellen Situationen eher Dialekt. Empirische Untersuchungen an objektsprachlichem Material (vgl. etwa das Erp-Projekt, dazu Niebaum/Macha 1999: 153-156) lassen erkennen, dass diese These in grundsätzlicher Hinsicht zutrifft. Ein genaueres Hinsehen bringt freilich zutage, dass die Gleichung 'eine Situation = eine Sprachvarietät' relativiert werden muss: Dauer und Dynamik, die jeder kommunikativen Interaktion eigen sind, lassen abrupte Wechsel zwischen Dialekt und Standardsprache ebenso zu wie fließende, sich in diversen Mischungen konkretisierende Übergänge.

Als ein vorläufiges Fazit aus den geschilderten Überlegungen lässt sich festhalten: Die regionalsprachlichen Zustände sind heutzutage so beschaffen, dass man bei ihrer Erforschung mit einem "offenen Sprechermodell" arbeiten sollte. Die traditionell veranschlagten Determinanten 'Sozialgruppe' und 'Situation' sind dabei in Rechnung zu stellen, sie müssen jedoch um Aspekte der individuellen Disposition von Sprechern erweitert werden. Welche Sprachformen jemand in kommunikativen Interaktionen auswählt und realisiert, lässt sich nur teilweise und mitunter nicht sehr erklärungskräftig auf die genannten Bestimmungsfaktoren zurückführen. Es ist deshalb notwendig, für jedes einzelne sprechende Individuum einen prinzipiellen 'Möglichkeitsraum' anzusetzen, mit dem ihm Optionen zwischen verschiedenen sprachlichen Varianten eröffnet sind. Heutzutage scheint es wichtiger denn je, sich als Dialektologe zu vergegenwärtigen, dass das, was ein Sprecher äußert, sowohl ein Ausdruck dessen ist, was er zu äußern imstande ist, als auch dessen, was er äußern will, dass also die feststellbare sprachliche Variation nicht zuletzt als 'Ergebnis von Handlungswahl' zu deuten ist. Die Beiträge eines Sammelbandes mit gleichlautendem Titel von Beate HennMemmesheimer (1998) stellen diesen bisher eher vernachlässigten Aspekt zurecht in den Vordergrund. Annelies Häcki Buhofer liefert dabei eine einleuchtende Darstellung der verschiedenen Einflussdimensionen, mit denen Sprechvarianz zu tun hat, und sie scheut sich erfreulicherweise auch nicht, eine Komponente individueller Sprachhandlungswahl zu betonen, die man als regelfixierter Sprachforscher außerordentlich ungern zur Kenntnis nimmt: die 'einmalige, unvorhersehbare, unberechenbare Seite der Identität' (vgl. Häcki Buhofer 1998: 66). Natürlich weiß ich auch, dass es den alten Satz gibt: "De singularibus non est scientia." Nur: Genaue Beobachtung, die auch dem Unerwarteten und dem Einzelnen 
gerecht wird, bedeutet keineswegs den Verzicht auf verallgemeinernde Musterbestimmung und damit ein Versinken im Forschungsatomismus. Im Gegenteil: Anhand eines individuenzentrierten Zugriffs lassen sich Typologien von Sprechern, Abbauhierarchien von Sprachmerkmalen und Sprachgebrauchs-Prognosen ebenso gewinnen wie Erkenntnisse über Dimensionen sprachlicher Variationsräume, über deren kognitive Organisation u.a.m.

\section{$3 \quad$ Mögliche Forschungsperspektiven}

Im Folgenden beziehe ich mich exklusiv auf Möglichkeiten, die mit einer sprecherdialektologischen Basis verknüpfbar sind, und nehme den Stand der Forschung zur Regionalsprachlichkeit zum Ausgangspunkt, so wie er im Jahrbuch des Instituts für Deutsche Sprache (Stickel 1997) kenntnisreich zusammengefasst ist. Wir haben im Blick auf Varietätenverwendung im deutschsprachigen Raum zwar informative Grobvermessungen demoskopischer Art, dieses Wissen ist jedoch weder vollständig noch hat es die nötige Tiefenschärfe. Sprachdemoskopie als Betrachtung aus der Vogelperspektive erbringt diskussionswürdige Aussagen, die im Sinne von Austins Granularitätstheorie als 'grobkörnigwahr' gelten können. Es bedarf freilich zusätzlich empirischer Analysen der Feinstruktur des Sprachgebrauchs. Alle ernstzunehmenden dialektologischen Arbeiten vermerken z.B. die permanente Wechselwirkung zwischen den verschiedenen Sprachvarietäten. Dieser Befund hat sein Pendant im sprechenden Individuum, insofern er sich in Form von Alternanz und Interferenz, von code switching, code shifting, code mixing etc. konkretisiert (vgl. Macha 2004). Bisher hat man sich dieses Faktums erst ansatzweise angenommen, eine interregional vergleichende Betrachtungsweise, die - bezogen auf den deutschsprachigen Raum - den eklatanten Verschiedenheiten in Sprachgebrauch und Sprachbewertung Rechnung trüge, gibt es bisher nicht, sie wäre aber dringend erforderlich. Denkbar und machbar sind dabei interregionale Vergleiche verschiedener Art. Warum nicht etwa einige selbständige Handwerksmeister pro Region aussuchen und deren sprachlichen Alltag mittels Sender aufzeichnen und analysieren? Warum nicht altershomogene Stichproben zugrunde legen und z.B. im ober-, mittel- und niederdeutschen Raum in Seniorenheimen Sprachverhalten explorieren? Warum nicht dasselbe in ausgewählten Kindergärten? Warum nicht - von einer anderen Seite her ansetzend - funktionale Erfordernisse konstant halten und die regionalsprachlichen Reaktionen darauf untersuchen? Hier gäbe es Beobachtungen zu machen, die sich mit Überlegungen zu einer 'Pragmatik nonstandardsprachlichen Sprechens' berühren würden (vgl. Bellmann 1994). Interessant scheinen mir in diesem Zusammenhang auch neuere Forschungszugriffe einer interaktionsbezogenen 'Gesprächsanalyse', in denen die Dialektproblematik eine nicht unwesentliche Rolle spielt.

\section{Das 'Posemuckel-Projekt'}

Despektierlich und zugleich liebevoll haben die InitiatorInnen ein anvisiertes Forschungsvorhaben das 'Posemuckel'-Projekt getauft, mit dem die Sprachgebrauchssituation in Norddeutschland transparenter gemacht werden soll. Neben einer Reihe von anderen wichtigen Ausrichtungsmarken sind bei diesem Vorhaben auch theoretische und methodischpraktische Fixpunkte aus der Sprecherdialektologie berücksichtigt worden. In Kooperation 
der Universitäten Hamburg (Ingrid Schröder), Bielefeld (Jan Wirrer), Bonn (Michael Elmentaler) und Münster (Jürgen Macha) wird eine 'interregional vergleichende Nonstandardforschung' in Angriff genommen, mit der die auf besondere Weise historisch gewachsenen Sprachverhältnisse Norddeutschlands neu beleuchtet werden sollen. Grob vereinfacht möchten wir Antworten auf - wenigstens - die folgenden sechs Fragen erhalten:

- Welche Form von Intendiertem Ortsdialekt (IOD) produziert man heute in Ortspunkten Norddeutschlands?

- Welche Form von Intendierter Standardsprache (ISS) wird heute in Ortspunkten Norddeutschlands gesprochen?

- Wie sieht in Ortspunkten Norddeutschlands eine 'standardnahe Umgangssprache' aus?

- Wie läuft in Ortspunkten Norddeutschlands sprachliches Handeln in und zwischen den Varietäten 'in vivo' ab?

- Wie beschreiben und bewerten Gewährspersonen in Ortspunkten Norddeutschlands ihren eigenen alltäglichen Sprachgebrauch?

- Wie beschreiben und bewerten Gewährspersonen in Ortspunkten Norddeutschlands ihre Sprachbiographie?

Zum gegenwärtigen Planungszeitpunkt zielt die Forschergruppe auf eine Erhebung in 54 Ortschaften Norddeutschlands, die eine Einwohnerzahl zwischen fünf- und zehntausend Einwohnern aufweisen. Die Orte sind durch einen Kriterienkatalog relativ vergleichbar gehalten, pro Ort werden dialektkompetente und nicht-dialektkompetente Gewährspersonen in verschiedenen settings, darunter in einer familienfestähnlichen 'in-vivo'Kommunikationssituation aufgenommen (vgl. die ausführliche Darstellung des Projekts im 'Korrespondenzblatt' des Vereins für niederdeutsche Sprachforschung 111, 2004: 31-33).

Am Ende dieses kleinen Beitrags möchte ich nur wenige knappe Hinweise auf den Fragehorizont geben, vor dem unsere Forschungsinteressen $\mathrm{zu}$ sehen sind. Die wissenschaftliche opinio communis setzt für Norddeutschland das Modell eines 'segregativen Sprachgebrauchs' bei denjenigen SprecherInnen an, die über die zwei Varietäten 'Intendierter Ortsdialekt' (IOD) und 'Intendierte Standardsprache' (ISS) verfügen. Es gibt demzufolge also 'alternative Wahlen': Entweder man spricht Mundart - wenn man sie denn überhaupt noch beherrscht - oder man spricht Hochdeutsch, beides wird im Gebrauch klar getrennt. Als Steuerungsprinzipien fungieren bei dieser Art von Bilinguismus die oben besprochenen dialektpragmatischen Größen. Wenn ich recht sehe, bestehen in puncto Varietätendistinktion durchaus Ähnlichkeiten zwischen Norddeutschland und der deutschen Schweiz. Auch die Eidgenossenschaft soll dadurch geprägt sein, dass "die Sprecherinnen und Sprecher Dialekt und Standardsprache strikt auseinander halten", so schreibt - allerdings mit fragendem Unterton - Helen Christen in einem Beitrag von 2000 (vgl. Christen 2000b: 245). Für Österreich, Süddeutschland und Teile der Mitte kursiert dagegen gemeinhin ein erheblich anderes Bild, bei dem ein 'integrativer' Sprachgebrauch, d.h. eine weitaus stärkere Mischung aus dialektalen und standardnahen Elementen angesetzt wird. Ob solche Makromodelle die 
Wirklichkeit angemessen wiedergeben, lässt sich erst dann entscheiden, wenn auf der Basis vergleichbar parametrisierter Untersuchungen interregionale Kontraste bzw. Übereinstimmungen nachgewiesen werden können. Den InitiatorInnen des 'Posemuckel'Projekts geht es nun darum, für die Sprachkonstellation in Norddeutschland genauer hinzusehen, als dies bisher vergleichend geschehen ist. Die Vielfalt der Variation im alläglichen Sprechen verlangt eine Nonstandardforschung, die zusätzlich zu den traditionell bewährten Zugriffen vor allem auch die Untersuchung von 'Sprache in vivo' praktiziert, also von Daten, die nicht bereits im Vorhinein, gewissermaßen vor ihrer Elizitierung kategorial ausgerichtet und purifiziert sind. Wie schwer ein solcher Anspruch in die Wirklichkeit umzusetzen ist, ahnt freilich jeder, der um die Wonnen und Wehen dialektologischer Feldforschung weiß.

\section{Literaturangaben}

Androutsopoulos, Jannis K./Ziegler, Evelyn (eds.) (2003): 'Standardfragen'. Soziolinguistische Perspektiven auf Sprachgeschichte, Sprachkontakt und Sprachvariation. Frankfurt/Main.

Auer, Peter/Gilles, Peter/Peters, Jörg/Selting, Margret (2000): "Intonation regionaler Varietäten des Deutschen. Vorstellung eines Forschungsprojekts". In: Stellmacher (ed.) (2000): 222-239.

Bausinger, Hermann (1967): "Bemerkungen zu den Formen gesprochener Sprache". In: Satz und Wort im heutigen Deutsch. Probleme und Ergebnisse neuerer Forschung. Jahrbuch 1965/66 des IDS. Düsseldorf: 292-312.

Christen, Helen (2000a): "Chamäleons und Fossilien. Forschungsperspektiven für die konsolidierte schweizerisch-alemannische Dialektologie". In: Stellmacher (ed.) (2000): 3347.

Christen, Helen (2000b): "Standardsprachliche Varianten als stilistische Dialektvarianten?" In: Häcki Buhofer (2000): 245-260.

Eichhoff, Jürgen (1977-2000): Wortatlas der deutschen Umgangssprachen. Bd. 1-4. Bern/München.

Gilles, Peter (2003): "Zugänge zum Substandard: Korrelativ-globale und konversationelllokale Verfahren". In: Androutsopoulos/Ziegler (ed.) 2003: 195-215.

Häcki Buhofer, Annelies (ed.) (2000): Vom Umgang mit sprachlicher Variation. Soziolinguistik, Dialektologie, Methoden und Wissenschaftsgeschichte. Tübingen und Basel.

Herrgen, Joachim/Schmidt, Jürgen E. (1985): "Systemkontrast und Hörerurteil. Zwei Dialektalitätsbegriffe und die ihnen entsprechenden Meßverfahren". ZDL 52: 20-42.

Herrgen, Joachim (2001): "Die Dialektologie des Deutschen". In: Auroux, Sylvain et al. (eds.): Geschichte der Sprachwissenschaften. Berlin/New York (= HSK 18.2): 1513-1535.

Hildebrandt, Reiner (1968): "Der deutsche Wortatlas als Forschungsmittel der Sprachsoziologie". In: Mitzka, Walther (ed.): Wortgeographie und Gesellschaft. Berlin: 149-169.

Löffler, Heinrich (1986): "Sind Soziolekte neue Dialekte? Zum Aufgabenfeld einer nachsoziolinguistischen Dialektologie". In: Schöne, Albrecht (ed.): Kontroversen, alte und 
neue. Akten des VII. Internationalen Germanistenkongresses. Göttingen 1985. Bd. 4. Tübingen: 232-239.

Macha, Jürgen (1983): "Die 'beliebteste' Mundart? Überlegungen zur Bewertung deutscher Dialekte". Rheinisch-Westfälische Zeitschrift für Volkskunde 28: 164-184.

Macha, Jürgen (1986): "Die Bedeutung individueller Variation. Zur Umwertung eines traditionellen Störfaktors". In: Schöne, Albrecht (ed.): Kontroversen, alte und neue. Akten des VII. Internationalen Germanistenkongresses. Göttingen 1985. Bd. 4. Tübingen: 300304.

Macha, Jürgen (1991): Der flexible Sprecher. Untersuchungen zu Sprache und Sprachbewußtsein rheinischer Handwerksmeister. Köln/Wien.

Macha, Jürgen (2004): "Regionalsprachliche Varietäten des Deutschen und ihre Dynamik". Der Deutschunterricht LVI: 18-25.

Mattheier, Klaus J./Wiesinger, Peter (eds.) (1994): Dialektologie des Deutschen. Forschungsstand und Entwicklungstendenzen. Tübingen.

Niebaum, Hermann/Macha, Jürgen (1999): Einführung in die Dialektologie des Deutschen. Tübingen. (= Germanistische Arbeitshefte 37).

Piirainen, Elisabeth (2003): "Areale Aspekte der Phraseologie: Zur Bekanntheit von Idiomen in den regionalen Umgangssprachen". In: Häcki Buhofer, Annelies/Burger, Harald/ Greciano, Gertrud (eds.): Flut von Texten - Vielfalt der Kulturen. Baltmannsweiler: 117128.

Piirainen, Elisabeth (2003): "Es ist noch nicht im Topf, wo's kocht. Zu Idiomen aus dem Raum der ehemaligen DDR". Niederdeutsches Wort 43: 202-219.

Schmidt, Jürgen Erich (1986): Die mittelfränkischen Tonakzente (Rheinische Akzentuierung). Stuttgart. (= Mainzer Studien zur Sprach- und Volksforschung 8).

Steinig, Wolfgang (1976): Soziolekt und soziale Rolle. Düsseldorf.

Stellmacher, Dieter (ed.) (2000): Dialektologie zwischen Tradition und Neuansätzen. Beiträge der Internationalen Dialektologentagung, Göttingen, 19.-21. Oktober 1998. Stuttgart. (= ZDL-Beiheft 109).

Stickel, Gerhard (ed.) (1997): Varietäten des Deutschen. Regional- und Umgangssprachen. Berlin/New York.

Wiesinger, Peter (1994): "Zum gegenwärtigen Stand der phonetisch-phonologischen Dialektbeschreibung". In: Mattheier/Wiesinger (eds.) (1994): 3-27.

Wiesinger, Peter (2000): "Die deutsche Dialektologie zwischen Tradition und Neuansätzen". In: Stellmacher (ed.) (2000): 15-32. 